\title{
Hormonal and Reproductive Factors are Associated With Chronic Low Back Pain and Chronic Upper Extremity Pain in Women-The MORGEN Study
}

\author{
Hanneke A. H. Wijnhoven, PhD,${ }^{\dagger}$ Henrica C. W. de Vet, $\mathrm{PhD},{ }^{\dagger}$ Henriëtte A. \\ Smit, PhD,* H Susan J. Picavet, PhD,* \\ *National Institute of Public Health and the Environment, Center for Prevention \\ and Health Services Research, Bilthoven; and ${ }^{\dagger}$ Institute for Research in \\ Extramural Medicine, VU University Medical Center, Amsterdam, The Netherlands.
}

\begin{abstract}
Study Design: Cross-sectional study of 11,428 women aged 20-59 years who were included in a postal questionnaire survey in the Dutch general population. Objective: To examine how hormonal and reproductive factors are associated with chronic low back pain (LBP) and chronic upper extremity pain (UEP) in women.
\end{abstract}

Summary of Background Data: Although LBP is suggested to be linked to hormonal and reproductive factors in women, results from previous studies are inconclusive. In addition, the association with chronic UEP is unknown.

Methods: Multivariate logistic regression models were used to examine associations between hormonal and reproductive factors (independent variables) and, respectively, chronic LBP, chronic UEP and combined chronic LBP/UEP. Associations were adjusted for age, level of education, working status, smoking, and overweight.

Results: Past pregnancy, young maternal age at first birth, duration of oral contraceptive use, and use of estrogens during menopause were associated with chronic LBP, while young age at menarche was associated with chronic UEP. Irregular or prolonged menstruation and hysterectomy were associated both with chronic LBP and chronic UEP. No positive associations were found for current pregnancy and number of children.

Conclusions: In adult women, hormonal and reproductive factors are associated with chronic musculoskeletal pain in general. Factors related to increased estrogen levels may specifically increase the risk of chronic LBP.

\section{Introduction}

Low back pain (LBP) is a major health and socioeconomic problem in Western society. ${ }^{[1]}$ In women, LBP is suggested to be linked to hormonal and reproductive factors, like (past) pregnancy, number of children, oral contraceptive use, estrogen use during menopause, and menstruation-related factors. However, results are inconclusive. The presence of LBP during pregnancy is widely reported, ${ }^{[2-5]}$ but whether or not pregnancy is a risk factor for LBP after pregnancy remains to be established. One study supports this association by showing that $10 \%$ of women with chronic back pain link its onset to a pregnancy. ${ }^{[6]} A$ higher number of live births is suggested to be associated with LBP. ${ }^{[6,7]}$ However, this may be related to childrearing and not to childbearing because this association was observed in both men and women. ${ }^{[7]}$

Many health care professionals believe there is an association between oral contraceptive use and LBP. ${ }^{[8]}$ However, scientific evidence for this association is scarce ${ }^{[9,10]}$ or not supportive. ${ }^{[11,12]}$ The positive association between the use of estrogens during menopause and LBP is well documented in observational studies. ${ }^{[13,14]}$ However, a preventive effect of estrogen-progestin treatment on back pain in slim premenopausal women with low bone mineral density was found 
Spine. 2006;31(13):1496-1502. (C2006 Lippincott Williams \& Wilkins

in a randomized controlled trial. ${ }^{[15]}$ The association between LBP and menstrual cycle remains unclear. Although back pain is a commonly described symptom of the premenstrual syndrome, ${ }^{[16]}$ no association between LBP and hormonal fluctuations during the menstrual cycle was found for female soccer players. ${ }^{[11]}$

Subjects with LBP often report other musculoskeletal complaints like upper extremity pain (UEP) as well. ${ }^{[17]}$ The tendency of clustering of chronic musculoskeletal complaints from different sites may reflect the existence of a more general musculoskeletal pain syndrome, ${ }^{[18,19]}$ which suggests that hormonal and reproductive factors associated with LBP may be related to musculoskeletal pain in other anatomic locations as well. One study ${ }^{[20]}$ of middle-aged women found a variety of musculoskeletal diseases associated with several reproductive factors: young maternal age at first birth (fibromyalgia); high maternal age at last birth; having $\geq 2$ children (pelvic joint syndrome); higher spacing time between childbirths (pelvic joint syndrome); metrorrhagia (rheumatoid arthritis); and sterilization (undefined arthritis, pelvic joint syndrome, and fibromyalgia). No associations were found for nulliparity, mean age at menarche, and menstrual cycling pattern.

In this article, data from a large-scale population-based study are used to examine associations between hormonal and reproductive factors and chronic LBP, chronic UEP and combined chronic LBP/UEP in women. The aim is to add new data to existing literature on the suspected involvement of these factors in the development of chronic LBP. Including UEP as an outcome parameter may help to understand the biologic mechanism between associations. Are they specific for LBP or not?

\section{Materials and Methods}

\section{Participants}

This investigation uses data from a general population-based study in the Netherlands: the Monitoring Project on Risk Factors for Chronic Diseases (MORGEN study). ${ }^{[19]}$ The research population of the MORGEN study consists of a random sex-age stratified sample of persons aged 20-59 years, who live in 3 towns (Amsterdam, Maastricht, Doetinchem) located in different regions in the Netherlands. The baseline assessment took place between 1993 and 1997. Municipal population registers were used as sampling frames. The response rate of the MORGEN study was $75 \% .{ }^{[19]}$ Cross-sectional data were available for 22,415 persons, of whom 12,283 were women. For the current analyses, 11,428 women were included; 855 were excluded because of missing data on the presence of LBP or UEP.

\section{Data Collection}

Data collection consisted of questionnaires sent by post and a physical examination. LBP and UEP (neck, shoulder, higher part of the back, or arms) were assessed using an adapted version ${ }^{[19]}$ of the Nordic questionnaire. For both LBP and UEP, first a screening question was used: Have you had trouble or discomfort with pain in the region of interest (LBP or UEP) during the past 12 months? The region of interest was illustrated by a drawing of a complete human figure indicating the area at issue in black. Those subjects with LBP or UEP were given a supplementary questionnaire in which was assessed, among other things, whether the subject had chronic LBP or chronic UEP during the past 12 months, lasting longer than 3 months. 
Spine. 2006;31(13):1496-1502. (C2006 Lippincott Williams \& Wilkins

All data used in these analyses were questionnaire-based, except data of weight and height to calculate body mass index (BMI), which were assessed by physical examination. Background information on subjects consisted of: age (categorized into 10-year intervals); working status (paid job or no paid job); level of education (primary school, junior vocational education, secondary vocational education, vocational colleges, university); current smoking status; and overweight (BMI $>25 \mathrm{~kg} / \mathrm{m}^{2}$ ). The following hormonal and reproductive factors were assessed: current pregnancy; past pregnancy; number of children; maternal age at first birth; ever used oral contraceptives; current use of oral contraceptives; duration of oral contraceptive use ( $\leq 5,5-15$, and $>15$ years); ever used estrogens or female hormones because of menopausal complaints; age at menarche; regularity of menstruation (unknown, irregular, regular cycle $\leq 26$ days, regular cycle of 27-29 days, regular cycle $>30$ days); and past hysterectomy.

\section{Statistical Analyses}

Multivariate logistic regression models were used to examine associations between hormonal and reproductive factors (independent variables) and, respectively, chronic LBP, chronic UEP, and combined chronic LBP/UEP (dependent variables). All models were adjusted for age, level of education, working status, smoking, and overweight. Separate analyses were performed with 3 mutually exclusive dependent variables: chronic LBP only, chronic UEP only, and combined chronic LBP/UEP. Each time, the reference category consisted of subjects without chronic LBP and chronic UEP. The odds ratios (ORs) and their confidence intervals (CIs) provide information of the direction, strength, and statistical significance of associations. The strength of the associations cannot be compared between determinants because of a lack of uniform units. The strength of associations within determinants can be compared between the 3 chronic musculoskeletal pain groups (chronic LBP, chronic UEP, and combined chronic LBP/UEP).

\section{Results}

In women ages 20-59 years, the prevalence of chronic LBP was $8.9 \%$, of chronic UEP, $14.6 \%$ and $12.6 \%$ had chronic LBP as well as chronic UEP. The proportion of women without chronic LBP or chronic UEP was $63.9 \%$ ( Table 1 ). Of those women with chronic LBP, chronic UEP, or both, around $80 \%$ reported that they had almost continuous complaints in the last year.

In Table 2 , associations between hormonal and reproductive factors and 3 chronic musculoskeletal pain categories (i.e., chronic LBP, chronic UEP, and combined chronic LBP/UEP) are shown. All background characteristics (i.e., older age, no paid job, lower level of education, current smoking, and being overweight) showed similar positive associations with chronic LBP, chronic UEP, and combined chronic LBP/UEP. Associations were strongest for combined chronic LBP/UEP.

Approximately $70 \%$ of the women had been pregnant, and $1.2 \%$ was pregnant during the study. Most women (74\%) had their first child between ages 20 and 30 years ( Table 1 ). Pregnancy during the study and the number of children were not associated with chronic LBP or chronic UEP. Being pregnant during the study was associated with no chronic UEP. Previous pregnancy was associated with chronic LBP, but not with chronic UEP or combined chronic LBP/UEP. A young maternal age at first birth, especially when younger than 20 years, was associated with chronic LBP and combined chronic LBP/UEP ( Table 2 ). 
Spine. 2006;31(13):1496-1502. (C2006 Lippincott Williams \& Wilkins

More than $85 \%$ of women had used oral contraceptives, and $56 \%$ used oral contraceptives during the study. Between ages 40 and 50 years, $8 \%$ of women had used estrogens, and between ages 50 and 60 years, this percentage was $25 \%$ ( Table 1 ). The ever used oral contraceptives and use of estrogens during menopause were both associated with chronic LBP and combined chronic LBP/UEP. For chronic LBP, the OR increased steadily with increased duration of oral contraceptive use. Current oral contraceptive use was weakly associated with chronic UEP and combined chronic LBP/UEP ( Table 2 ).

Most women (69\%) were 12-14 years old when they had their menarche. There was $18 \%$ percent of women who reported an irregular menstruation cycle, and $9 \%$ had a prolonged ( $>30$ days) menstrual cycle. In the total study population, $11 \%$ of the women had undergone a hysterectomy ( Table 1 ). Young age at menarche ( $\leq 11$ years) was associated with chronic UEP and combined chronic LBP/UEP, but not with chronic LBP. An irregular or prolonged menstrual cycle was associated with chronic LBP, chronic UEP and combined chronic LBP/UEP, although not all associations were statistically significant. Past hysterectomy was associated with chronic LBP, chronic UEP, and combined chronic LBP/UEP.

\section{Discussion}

This study showed that hormonal and reproductive factors like irregular or prolonged menstrual cycle and hysterectomy were associated both with chronic LBP and chronic UEP in women. Estrogen-related factors like past pregnancy, young maternal age at first birth, duration of oral contraceptive use, and use of estrogens during menopause were specifically associated with chronic LBP, and not with chronic UEP. Young age at menarche was only associated with chronic UEP, and no positive associations were found for pregnancy during the study and number of children. All associations were adjusted for age, level of education, working status, smoking, and overweight.

Some remarks should be made when interpreting the results of this study. First, self-report assessment of chronic musculoskeletal pain, and hormonal and reproductive factors may have resulted in nondifferential misclassification of data because of recall bias, which usually results in an underestimation of associations. ${ }^{[21]}$ There are no obvious reasons to assume differential misclassification because of recall bias. Factors especially like duration of oral contraceptive use, age at menarche, and regularity of menstruation may be subject to nondifferential recall bias.

Second, the cross-sectional nature of the study does not allow disentanglement of cause and effect. Hormonal and reproductive factors may be either risk factors for chronic musculoskeletal pain, or, as suggested by Skomsvoll et al, ${ }^{[20]}$ musculoskeletal pain may alter reproductive function in women. In addition, psychologic, social, or behavioral factors could explain the association between reproductive factors and musculoskeletal pain. We did adjust our analyses for vitality and mental health (subscores of the RAND-36 questionnaire) ${ }^{[22,23]}$ and physical activity, ${ }^{[24]}$ and found no effect on the associations reported here. Because these factors were not assessed in the first and, for vitality and mental health, also second year of the survey, they were not included in the final analyses.

The third remark concerns a potential nonresponse bias. Although it is possible that musculoskeletal pain may be more prevalent among nonresponders, it is not likely that nonresponse bias has affected the association between hormonal and reproductive factors and chronic musculoskeletal pain because the MORGEN study 
is a general health survey not primarily aimed at examining this association. ${ }^{[19]}$ The final remark concerns the relatively small ORs that are statistically significant because of the large sample size of our survey. However, although the ORs are small, the impact in terms of an extra number of cases may be considerable because of the high prevalence of risk factors and chronic pain complaints.

Pregnancy during the study was not found to be associated with chronic LBP. This result may be because of the fact that the pregnant population in this study may be too small to detect statistically significant associations, and LBP may not (yet) be chronic in women who became pregnant only a few months ago. Past pregnancy was associated with chronic LBP, which supports the finding that $10 \%$ of women with chronic back pain link its onset to pregnancy. ${ }^{[6]}$ Although the etiology and pathophysiology of LBP during pregnancy are poorly understood, one plausible hypothesis is that causes of LBP in pregnancy are multifactorial (i.e., biomechanical stress on ligaments and joints, muscle fatigue, and the effect of the hormone relaxin on joint laxity), with $\geq 1$ factors dominating in an individual case. ${ }^{[25]}$

Unlike results from other studies, ${ }^{[6,7]}$ no association was found between the number of children and chronic LBP. A previous population-based survey ${ }^{[7]}$ found a linear association between the number of live births and chronic LBP, even after adjusting for age, BMI, smoking, and car ownership as an indicator of social class in both men and women. Also in pregnant women, the number of live births was associated with LBP. ${ }^{[5]}$ In our study, unadjusted analyses show a linear association between the number of children and chronic LBP and chronic UEP (data not shown). This association disappeared mainly after adjustment for age and to a lesser extent after adjustment for level of education. Results from our study suggest that in the general population, there is no independent association between the number of children and chronic LBP or chronic UEP.

Young maternal age at first birth, especially younger than 20 years, was associated with chronic LBP and combined chronic LBP/UEP, suggesting that this may be a risk factor for chronic LBP in women. Stratified analyses show that this association is found in all age groups (data not shown). In another populationbased survey among women, ${ }^{[7]}$ younger age at first pregnancy was also associated with a higher prevalence of ever having LBP. Of pregnant women, 2 studies show positive associations between younger maternal age and a higher prevalence of LBP or more pain intensity, ${ }^{[3,26]}$ while another study does not find such associations. ${ }^{[5]}$ Explanations for a possible relationship between young maternal age and chronic LBP are unknown.

The ever used oral contraceptives appeared to be specifically associated with chronic LBP, and not with chronic UEP. The validity of this finding was strengthened by the linear association between duration of oral contraceptive use and LBP, which was even stronger in women aged 50-60 years (data not shown). In 1995, Brynhildsen et $a{ }^{[8]}$ reported that many health professionals believe that there is an association between oral contraceptive use and LBP, despite the lack of scientific evidence. Although oral contraceptive use was not associated with LBP in young women, ${ }^{[11]}$ pregnant women, ${ }^{[5,27]}$ and no association was found with referral to hospital for back disorders in young women, ${ }^{[12]}$ the positive association found this study as well as in other general population studies ${ }^{[9,10]}$ stresses the need for additional research on the association between oral contraceptive use and chronic LBP.

The ever used estrogens during menopause was associated with chronic LBP and combined chronic LBP/UEP. Previous studies show a positive relationship between 
postmenopausal estrogen use and back pain. ${ }^{[13,14]}$ Musgrave et a ${ }^{[14]}$ analyzed data from 7209 postmenopausal women, and found a positive association between estrogen use and back pain after adjusting for vertebral fractures, age, smoking history, BMI, exercise status, parity, diabetes mellitus, and arthritis. Residual confounding was discussed; estrogen users may be less tolerant of back symptoms or women may begin estrogen therapy, erroneously believing that their back pain is related to osteoporosis. However, the association between estrogen use and back problems remained even after excluding women with vertebral fractures and/or women believing that they were osteoporotic. ${ }^{[14]}$ In contrast with these findings, a diminishing effect of estrogen-progestin treatment on nighttime back pain and overall back disability was found in slim premenopausal women with low bone mineral density. ${ }^{[15]}$ However, this beneficial effect on back pain was not found in healthy early postmenopausal women, ${ }^{[28]}$ suggesting that use of estrogens has a potential beneficial effect on back pain in slim premenopausal women with low bone mineral density, while it negatively affects LBP in postmenopausal women.

We found menarche at young age ( $\leq 11$ years) and an irregular or prolonged menstrual cycle to be associated with chronic UEP, chronic LBP and combined chronic LBP/UEP (although not all associations are statistically significant), suggesting that these factors may be associated with chronic musculoskeletal pain in general. Although LBP is positively correlated with menstruation in women $^{[6]}$ and back pain is a common symptom of the premenstrual syndrome, ${ }^{[13]}$ there are only a few studies describing the association between menstrual pattern and musculoskeletal disorders. One population-based study of 40-42-year-old women found no association between the mean age of menarche or menstrual cycling pattern and musculoskeletal diseases. ${ }^{[20]}$ Another study of 152 women with persistent pelvic pain and pelvic joint instability following parturition showed that mean age at menarche was significantly lower than expected in the general population. ${ }^{[29]}$ Yet another study showed that LBP during pregnancy was not related to mean age at menarche or irregular menstrual cycle but was associated with amenorrhea. ${ }^{[5]}$ Our data and data from previous studies ${ }^{[20,5]}$ do not support an association between menstrual cycling pattern or age at menarche and LBP specifically, but the relationship found with UEP indicates that there may be an association with chronic musculoskeletal pain in general, which needs further study.

We found that hysterectomy was associated with chronic LBP, chronic UEP, and combined chronic LBP/UEP, suggesting that hysterectomy may be associated with chronic musculoskeletal pain in general. To our knowledge, no previous studies on this association were performed. As pointed out by Ostensen and Schei, ${ }^{\left[{ }^{[0]}\right.}$ the significant association between musculoskeletal symptoms and gynecologic dysfunction raises the question as to whether a history of disorders of the genital tract predisposes for the development of musculoskeletal diseases. It is equally possible that that endometriosis or chronic pelvic pain (as potential indications for hysterectomy) and chronic musculoskeletal pain may both be part of an overarching pain problem.

Past pregnancy, young maternal age at first birth, duration of oral contraceptive use, and use of estrogens during menopause all show associations with chronic LBP, but not with chronic UEP. Although there is much debate about the exact biologic basis for these associations, it is generally believed that variation in women's estrogen levels plays an important role. One theory is that increased estrogen levels result in increased laxity of joints and ligaments. This increased laxity then leads to LBP. ${ }^{[3,14]}$ It has been suggested that the hormone relaxin, a hormone of pregnancy, causes this joint laxity in pregnant women. ${ }^{[8]}$ Because 
Spine. 2006;31(13):1496-1502. (C2006 Lippincott Williams \& Wilkins

relaxin levels have been increased in women using oral contraceptives, ${ }^{[10]}$ it is also suggested that relaxin may provide the missing key for understanding the association between oral contraceptive use and LBP. ${ }^{[9,31]}$ However, recent studies have found that increased peripheral joint laxity in pregnant women was not associated with increased serum relaxin levels ${ }^{[32,33]}$ or estradiol levels. ${ }^{[32]}$ Furthermore, oral contraceptive use was not associated with laxity measurements at the knee or hand, ${ }^{[34]}$ suggesting that oral contraceptive use may affect laxity of pelvic ligaments, resulting in LBP but not of peripheral ligaments in the knee or hand. This result may explain why past pregnancy, oral contraceptive use, and estrogen use during menopause are associated with chronic LBP but not with chronic UEP.

Pregnancy (past), oral contraceptive use, and use of estrogens during menopause may result in higher estrogen levels, resulting in an increased laxity of joints and ligaments through increased relaxin, resulting in chronic LBP, but not chronic UEP. However, this mechanism may not be that simple, and more research is needed. It is noteworthy that contrary to the supposed negative effects of estrogen on chronic LBP through an increased laxity of joints, estrogen is suggested to have a positive effect on pain suppression in general. ${ }^{[35,36]}$ In addition, a positive effect of estrogens on LBP is found in slim premenopausal women with low bone mineral density. ${ }^{[15]}$ Apparently in women from the general population between 20 and 59 years of age, the negative effects of estrogens on chronic LBP are stronger than these potential positive effects.

\section{Conclusions}

Hormonal and reproductive factors like an irregular or prolonged menstrual cycle and hysterectomy are associated both with chronic LBP and chronic UEP, suggesting that these factors are associated with musculoskeletal pain in general. Factors related to increased estrogen levels like (past) pregnancy, young maternal age at first birth, duration of oral contraceptive use, and use of estrogens during menopause may specifically increase the risk of LBP. More research is needed to examine these associations and unravel biologic explanations.

\section{Acknowledgements}

The authors thank the epidemiologists and field workers of the Municipal Health Services in Amsterdam, Doetinchem and Maastricht for their contribution to the data collection for this study. The Project Steering Committee consisted of Dr. H.B. Bueno de Mesquita, Prof. Dr. J.C. Seidell, Dr. H.A. Smit, and Dr. W.M.M. Verschuren. The authors also thank A. Blokstra, MSc, P.E. Steinberge, MSc, and A.W.D. van Kessel, MSc, for data management, and A. Jansen and J. Steenbrinkvan Woerden, MSc, for logistic support.

\section{Reprint Address}

Address correspondence and reprint requests to Susan J. Picavet, PhD, National Institute of Public Health and the Environment (RIVM), Center for Prevention and Health Services Research (PZO, pb 101), PO box 1, 3720 BA Bilthoven, The Netherlands; E-mail: susan.picavet@rivm.nl 